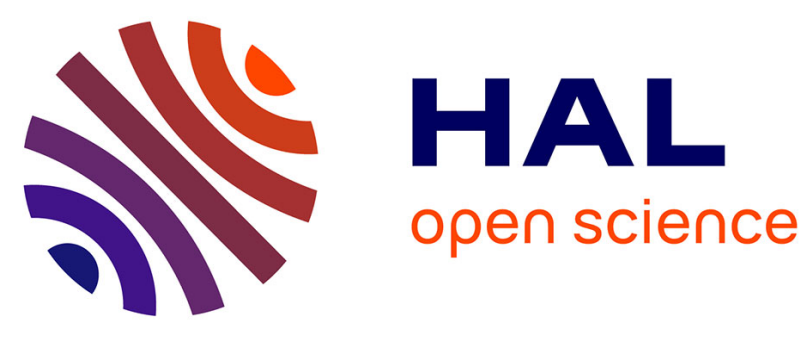

\title{
Breathing Friend: Tackling Stress Through Portable Tangible Breathing Artifact
}

Miroslav Macik, Katerina Prazakova, Anna Kutikova, Zdenek Mikovec, Jindrich Adolf, Jan Havlik, Ivana Jilekova

\section{To cite this version:}

Miroslav Macik, Katerina Prazakova, Anna Kutikova, Zdenek Mikovec, Jindrich Adolf, et al.. Breathing Friend: Tackling Stress Through Portable Tangible Breathing Artifact. 16th IFIP Conference on Human-Computer Interaction (INTERACT), Sep 2017, Bombay, India. pp.106-115, 10.1007/978-3319-68059-0_6. hal-01679770

\section{HAL Id: hal-01679770 \\ https://hal.inria.fr/hal-01679770}

Submitted on 10 Jan 2018

HAL is a multi-disciplinary open access archive for the deposit and dissemination of scientific research documents, whether they are published or not. The documents may come from teaching and research institutions in France or abroad, or from public or private research centers.
L'archive ouverte pluridisciplinaire HAL, est destinée au dépôt et à la diffusion de documents scientifiques de niveau recherche, publiés ou non, émanant des établissements d'enseignement et de recherche français ou étrangers, des laboratoires publics ou privés. 


\title{
Breathing Friend: Tackling Stress through Portable Tangible Breathing Artifact
}

\author{
Miroslav Macik ${ }^{1}$, Katerina Prazakova ${ }^{2}$, Anna Kutikova ${ }^{2}$, \\ Zdenek Mikovec ${ }^{1}$, Jindrich Adolf ${ }^{1}$, Jan Havlik ${ }^{1}$, and Ivana Jilekova ${ }^{1}$ \\ 1 Faculty of Electrical Engineering, \\ 2 Faculty of Architecture, \\ Czech Technical University in Prague, \\ Prague, Czech Republic \\ macikmir@fel.cvut.cz
}

\begin{abstract}
We present Breathing Friend - a portable and tangible device that uses haptic interaction to unobtrusively stimulate mindful breathing as efficient stress coping method. Its design is optimized for holding in user's hands. By changing its shape, it sends haptic signals to the user. Several studies were conducted where we explored and verified the form factors of the artifact, interaction methods, therapeutic effect, fitting to everyday life, and influence on the breathing pattern.
\end{abstract}

Keywords: Stress; industrial design; mindful breathing; tactile device.

\section{Introduction}

Long-term stress becomes a serious issue. It negatively influences the cognitive, emotional and physical well-being of many individuals. Stress can increase the probability of health problems like various cardiovascular diseases [12], hypertension, obesity, and depression [8]. An individual can deal with a stressful situation at any time and any place [13].

Traditional ways to tackle stress without medication include meditation, deep breathing, and relaxation. From the variety of breathing techniques, we selected the mindful breathing meditation that is used as a stress coping method [10]. It requires sophisticated training to tackle stress, or it is conducted with the help of a therapist.

Feijs et al. in [4] describe the relationship between stress, heart rate variability (HRV), and breathing. Breathing affects the heart rate through an effect called respiratory sinus arrhythmia. Training of breathing pattern can increase the HRV which lowers the stress level. Authors also suggest employing a portable device as it offers an opportunity to find a quiet place for relaxation. They recommend that such a device should not resemble computer or other ICT used for stressful work activities.

In our research, we investigate a possibility to design such alternative device that would efficiently stimulate mindful breathing. We introduce Breathing Friend $(B f)$ - a portable and tangible device that does not resemble ICT. Instead 
of already overloaded visual or auditory modality, it uses haptic interaction to discreetly and unobtrusively stimulate mindful breathing.

\section{Related work}

We focus on approaches aiming at controlling the breathing patterns. Particular attention is put to solutions exploiting haptic interaction modality.

Applications exist that help individuals with mindful breathing, e.g. Breathe App for Apple Watch [7], Spire [14]. However, those applications require the use of complex ICT devices which are identified as an inherent source of stress stimuli [5].

In [1], Rhawi et al. introduce a personalized biofeedback game that trains subjects to relax while playing a game. The game difficulty increases if the subject's breathing rate differs from a prescribed target rate. Validation on small group suggests that the game helps to acquire deep breathing skill and reducing arousal in following stress-inducing tasks.

Stahl et al. in [15] present a somaesthetic approach to support meditative bodily introspection by using light and heat modalities. Subjects report on an increased awareness of their breathing. Sato et al. [11] propose a music presentation system that uses listener's respiration information to change replay speed to minimize the difference between the target of and observed (listener?s) respiration phase. Paro [17] is a therapeutic robot resembling baby seal equipped with touch sensors allowing physical interaction. However, it is not intended to support mindful breathing.

Alonso et al. in [2] present Wigo, an embedded tangible device that aims to contribute to stress reduction. While under stress, people tend to make irrelevant gestures with physical objects (i.e. a pen), the intensity of such an activity increases when individuals are given a mentally demanding task. The stresscoping method is based on detection of stress by measuring irrelevant gesture activity - wiggling and making the movement increasingly more difficult. In [3], they present three devices (including Wigo) that show various kinds of haptic feedback to support stress reduction. The other two methods employed following activities: rolling over acrylic marbles (Marmoro) and squeezing (Squeeze-it).

Analysis of the related work indicates that tackling stress by stimulating mindful breathing can be successful $[4,1,11]$. Other research $[15,2,3]$ shows that tactile stimulation of hands can be used for stimulating mindful breathing.

\section{Design}

During the design, we had in mind that the final form should be visually and haptically pleasing [18], not inducing stigmatization [9], and providing efficient haptic signals to stimulate breathing.

Form factors. The design process started by molding several shape prototypes from clay and consequently from plaster (for fine surface finishing). The 


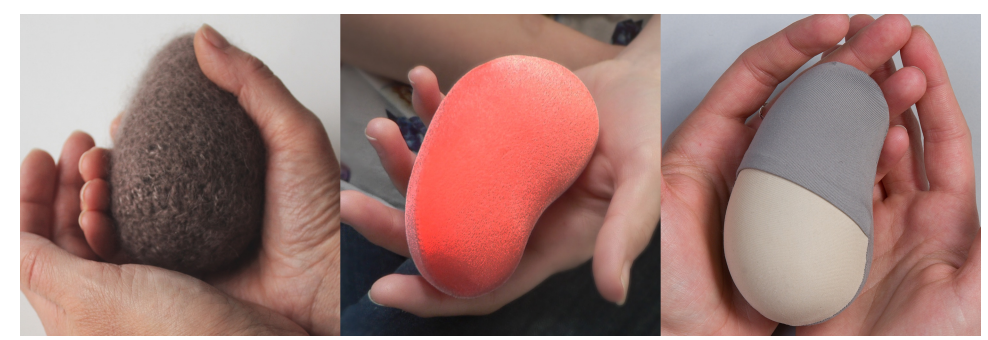

Fig. 1. Evolution of coating: knitted from wool (left), sewed from an elastic material (middle), easily exchangeable pattern sewed from an elastic material (right).
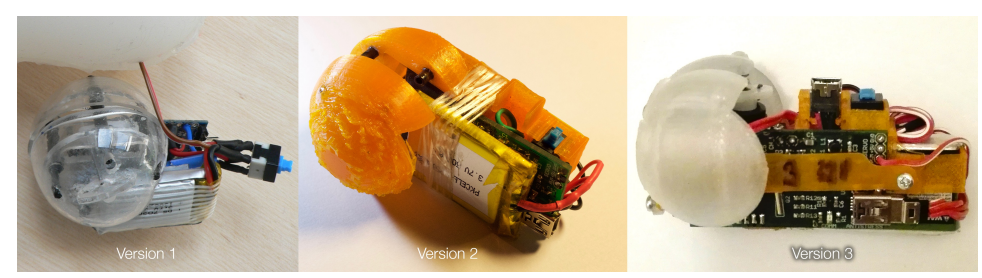

Fig. 2. Evolution of $B f$ electro-mechanical engine: the original handcrafted prototype (left), 3D printed + linear movement (center), 3D printed + strong servo (right).

final shape resembles an embryo evoking something alive that supports positive affect for users without elimination of public use. We conducted an informal study focused on the perception of the $B f$ form. The most promising embryonic shape resembled a small animal like a hamster or a tiny bird. The final prototype was created from silicone which has similar tactile properties as human skin and provides enough flexibility for breath-like motion. It is hollow to allow insertion of an electro-mechanical engine to provide tactile sensation of breathing.

Surface and personalization. Silicone is flexible but sticky. Therefore a coating was introduced. The first version was knitted from wool (see Fig. 1 left). The evaluation showed that it is too warm and fluffy, and can discourage some participants (see 4.1). Next version was made from an elastic material and had happy and bright colors (see Fig. 1 middle). The final version used a similar material, but more decent colors and different pattern (see Fig. 1 right). We also made the coating exchange easier for the user to support personalization.

Interaction and breathing pattern. The interaction with $B f$ starts by its activation via motion detection. The electro-mechanical engine in $B f$ starts cyclic inflation and deflation phases. The inflation phase is faster than the deflation phase. As therapist suggest (see 4.2) breathing rate gradually slows down from $15 \mathrm{brpm}$ (breath rate per minute) to $6 \mathrm{brpm}$. The electro-mechanical engine is placed in a silicone enclosure to provide distinct and smooth mechanical movement. Fig. 2 shows individual development versions of the engine. 
Implementation. An off-the-shelf high torque servo mechanism was used as an actuator spreading out two hemispheres. We developed three versions with gradually increasing servo mechanism torque and reliability as depicted in Fig. 2. Since version 2, we used a custom electronic board with a microcontroller (ATmega328), servo-control, voltage booster, Li-Po charging, accelerometer, realtime clock, 8M flash memory, and FTDI USB serial interface. Most components are 3D-printed to simplify manufacturing and assembly.

\section{Evaluation}

We conducted several formative evaluations focused on various aspects of human interaction with the device and its effect on the stress: first impression, the way of holding the device; therapeutic effect; how it fits into everyday life. We also investigated the existence of an effect on the breathing pattern.

\subsection{Form factors evaluation}

In this qualitative evaluation, we focused on the subjective perception of the final shape and wool coating (see Fig. 1 left). Based on the consultation with therapists and the study [6], in this evaluation, we focused on women returning to work after maternity leave as they are highly endangered by stress.

Participants. We recruited five female with kids and full/part time jobs (3 full-time, 2 part-time), average age $39.8(M I N=36, M A X=43, S D=2.6)$.

Procedure. We conducted five semi-structured interviews (about 45 minutes each), which took place in an informal, non-lab setting. First, we asked participants about their stress coping strategies. Second, we provided the participants with the $B f$ prototype and observed their reactions.

Results. All participants acknowledged that stress is a non-trivial thing and that it should be prevented or handled. Two said that prevention is the best form of the stress coping strategy (P4, P5). One of them had serious health problems caused by stress in the past (P4).

Two participants stated they would not feel to be ashamed while tackling stress by $B f(\mathrm{P} 4, \mathrm{P} 5)$; however, they indicated that $B f$ should be discreet (P4, $\mathrm{P} 5)$. Three participants stated that it is not acceptable to be in stress in public (P1 - P3) and that they feel sorry for their family members, who sometimes have to deal with a stressed out person (P2, P3).

All participants identified some animal form in the $B f$ (mouse, little hedgehog, mole, kitty). Some commented on the shape (bean, potato, sausage, kidney) and live-like movements (breathing child, snoring guy). Except for P5, all participants expressed positive attitude. P1 stated that it feels like "somebody is holding her hand". Participants used their dominant hand or both hands to hold it. Some were careful and gentle (P1, P2, P4) while others were trying to squeeze the $B f(\mathrm{P} 3, \mathrm{P} 5)$. Some participants stated that the $B f$ is too big to be handled discreetly; however, they did not experience difficulties in handling it. The movement reminded them of heart beating (P3 - P5), slow flow of fluid (P1), 
breathing (P5). Most found the movement pleasant (P1 - P4), one unpleasant (P5). They mentioned that they would prefer more cheerful and bright colors. They described the fuzzy appearance as "suitable for the winter months", most would prefer something "not so warm".

This evaluation led to the design of more appropriate coating (see Fig. 1) and more reliable electro-mechanical engine to provide stronger movement sensation.

\subsection{Potential therapeutic effect}

In this study, we focused on the potential of $B f$ to serve as a therapeutic aid. We interviewed therapists to gain insight into the usage of breathing in their praxis and to estimate the usefulness of the $B f$ for their clients.

Participants. Six therapists were inquired. Their specialization varied from a medical doctor, psychologist to yoga lecturer. All use therapeutic breathing for the training of self-awareness, and stress prevention and management.

Procedure. A semi-structured interview focused on their profession and characteristics of their patients was followed by presentation of the $B f$ device. They were asked to try it and express their opinions and suggestions. Afterward, they were asked to comment on the breathing pattern of the $B f$.

Results. Therapists described the $B f$ as very calming, useful for sensing mindful breathing, better focusing on breathing itself, and for a faster education of breathing patterns. Their methods spring from both research and ancient meditation techniques. Therapists teach patients to slow down their breathing to a particular frequency. P3 stated: "Tactile input is a promising method how to educate mindful breathing faster and perform it without direct contact with a therapist". This finding is in correlation with our subsequent literature overview. E.g. Vaucelle et al [16] states: "Touch is fundamental to our emotional well-being. Medical science is starting to understand and develop touch-based therapies for autism spectrum, mood, anxiety, and borderline disorders".

They suggested potential use also as a tool for rehabilitation. P4 suggested: "It could be utilized after intubation of children". Most therapists suggested decreasing breathing frequency. Based on these results, we configured the electromechanical engine to provide tactile sensation with a gradually decreasing rate from 15 down to a preset value of $6 \mathrm{brpm}$.

\subsection{Fitting into everyday life}

We explored how the $B f$ fits into everyday life activities of potential users.

Participants. We recruited four participants (three female), average age $36.75(M I N=34, M A X=39, S D=2,22)$ without previous experience with the $B f$. P1 works as a project manager; $\mathrm{P} 2$ as babysitter coordinator; $\mathrm{P} 3$ as manager in a small restaurant; $\mathrm{P} 4$ as a waiter in a teahouse.

Procedure. We conducted a fourteen-day diary study. Each participant was provided with a $B f$ device, a charging cable and a paper diary with a short questionnaire for each event of use. Improved version 3 of electro-mechanical 
engine was used for P3-4 (see Figure 2). They were instructed to fill the diary at least daily and to charge the $B f$ overnight. In the end, we conducted semistructured post-test interviews.

Results. The first impressions were diverse. P1 described the $B f$ as "...something for a massage?"; P2 as a bean-shaped object with a movement that reminds her of heart contractions; P3 described the $B f$ as an animal and highlighted its cuteness; P4 as a pink kidney.

For P1, the study was limited to one week due to a technical issue. She used the $B f$ seven times. She preferred to use it at work or home; "I used it when I feel to have time for myself," she mentioned. In the post-test interview, she highlighted the irregular movement caused by the technical issue.

For P2, twelve usages were recorded. She did not use it at work but at home when falling asleep ("I liked that I fell asleep."). She mentioned that it is not comfortable for her to breathe synchronously with it.

P3 used the $B f$ ten times, always at home in the evening for around 10 minutes. She mentioned the lack of free time as the reason for not using it. The most common positive comments in the diary were the movement of inflation, regularity of the motion, surface, moment to myself, contribution to relaxation. The negative comments were the whirring sound ( "Once I wanted to use it in a tram but, because of the whirr I could not."), fast battery discharging ("I would like to use it longer while watching a movie, but the battery discharged after 10 minutes.") and difficult synchronization of breath with $\mathrm{Bf}$ ("It is difficult for me to synchronize my breathing, it is not clear when to breathe in.").

For P4, only four usages were recorded. He used the $B f$ at home or in the teahouse, where he works. He mentioned serious work problems as the reason for not using it for the second week of the study. The negative comments were problems with charging, a bad response to the on/off switch, and the sound: "At the beginning I disliked the sound, but later I got used to it. In the end, I used it just while listening without touching. It makes the breathing pattern more clear than the movement".

Participants used $B f$ mostly once a day. There were positive outcomes; P2 used $B f$ for calming before sleep, P2, P3 described the use as "I like the time for myself.". All of them liked the surface material. P2 described that the pre-set breathing rate was too fast. Technical issues reported (discharging, unwanted activation) had an adverse impact on the use. However, only P2 contacted us to get technical help.

\subsection{Effect on the breathing frequency}

To evaluate the effect of $B f$ on breathing pattern we conducted a quantitative study.

Participants. We recruited 16 participants ( 5 female), average age 28.5 $(S D=9.4, M I N=22, M A X=61)$.

Apparatus. The experiment was conducted in usability lab decorated to support relaxation. It was equipped with a comfortable chair, footstool, and $\mathrm{TV}$ set, where an unintrusive movie clip was played in a loop. A prototype of 
$B f$ device was at disposal. There was a standard camera directed on the upper part of the participant's body. $B f$ device recorded its breathing frequency using internal memory and an RTC chip.

Experiment design. The experiment was one factor (two levels) withinsubject design. The independent variable was the way of interaction with the $B f$. Main measures were breathing frequency of participant and the $B f$ (brpm). For statistical evaluation, we used $95 \%$ confidence intervals.

Procedure. Participants were comfortably seated and the $B f$ was introduced as a device for tackling stress. Before the experiment started, they were sitting for at least 5 minutes. The experiment was divided into two blocks where the brpm was measured. In the Block 1 we instructed the participants to relax avoiding any instruction regarding the breathing. Participants were left alone in the room for approximately 10 minutes. After the first block, the subjective evaluation (at least 5 minutes) by means of a questionnaire (Likert scale 1-5) was performed. Then the Block 2 was started. Before the start, the participants were instructed to relax again and try to follow the simulated breathing of the $B f$ device. After the second block, the subjective evaluation was performed.

Results. The breath frequency was calculated from the video signal by application of a set of filters and an autocorrelation function. The average values with $95 \%$ confidence intervals are depicted in Fig. 3. In the Block 2, participants breath frequency was much closer to $B f$ than in Block 1 . This is clearly visible in the first 200 seconds (indicated by red vertical dashed line A). Then the frequency stagnated and from $300^{\text {th }}$ second (indicated by red vertical dashed line B) it even started to increase. The results show that during the Block 1 the breath frequency almost did not change, oscillating around 15-16 brpm. This is in contrast to values in Block 2, where the frequency clearly dropped down from $14.1 \mathrm{brpm}$ to below $9.6 \mathrm{brpm}$.

The subjective evaluation shows that more participants tried to voluntarily breathe in Block 2. However, the feeling of relaxation was much lower during

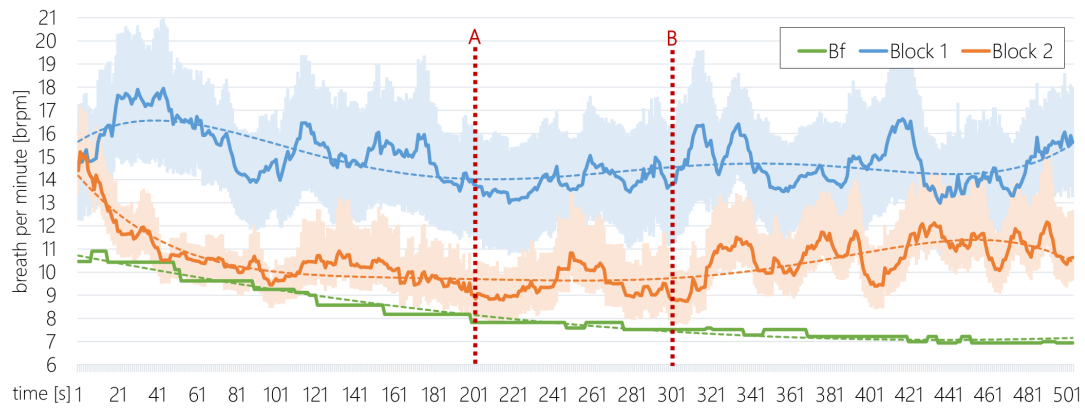

Fig. 3. Breath frequency and $95 \%$ confidence intervals of quantitative evaluation. The blue curve represents values from Block 1, orange curve values from Block 2, and green curve $B f$ frequencies (quantization error is caused by the 1s accuracy of RTC chip). 


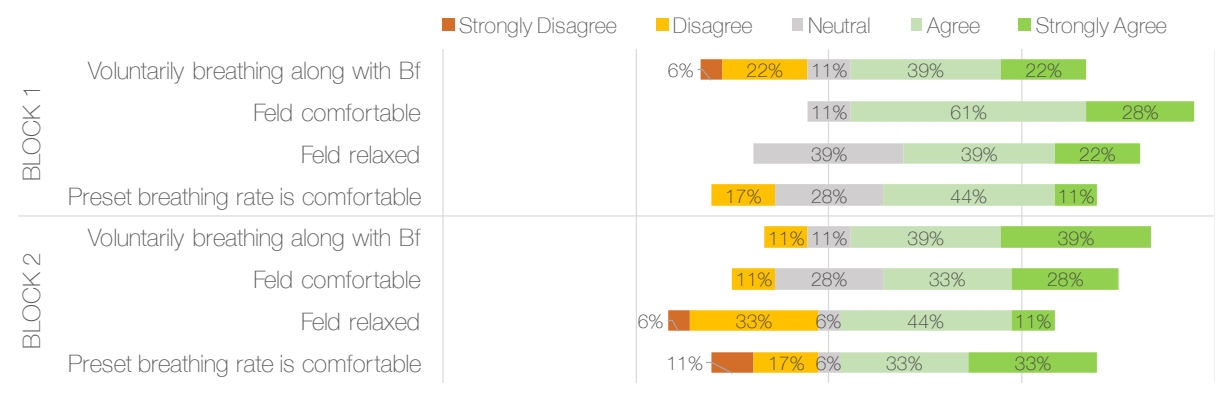

Fig. 4. Subjective evaluation of $B f$. Likert scale scores for both Blocks usage of $B f$.

Block 2 (39\% not relaxed vs. $0 \%$ in Block 1). This can be explained by the inability to breathe as slowly as the $B f$, as eight participants reported problems with adaption to low frequencies of $B f$. The surface of $B f$ was perceived as pleasant (83\%) and $94 \%$ agreed on the comprehensibility of $B f$ use. The noise of the electro-mechanical engine was disturbing for $69 \%$ of participants.

\section{Conclusion and Future Work}

We successfully designed a portable, tangible artifact that shows the ability to stimulate mindful breathing as a stress coping method. Evaluations indicate that it is possible to rely on haptic interaction only while avoiding the necessity to employ ICT like devices and audio-visual interaction.

Our evaluations showed the need for personalization of breathing pattern. Gradual adjustment of target breathing rate could eliminate discomfort during the attempt to slow down the breathing rate. The electro-mechanical engine should be as silent and as fluent as possible as the sensitivity of users to these aspects is very high. The user acceptance of the artifact can be significantly increased by introducing customization of the surface; thus the variability and ease of exchange of the coating are essential.

The future research should focus on finding an optimal course of breathing rate adjustment to reduce personal discomfort while preserving target rates suggested by therapists. Other research direction can focus on the usage of such artifact within the therapy session. Yohanan and MacLean in [18] leverage touch as an important part of emotional communication. Further research can focus on emotional communication potential of devices like $B f$.

\section{Acknowledgments}

This research has been supported by the TACR research program TE01020415, by internal CTU grants SGS16/236/OHK3/3T/13 and SGS17/183/OHK3/3T/13. 


\section{References}

1. Rami G Al Rihawi, Beena Ahmed, and Ricardo Gutierrez-Osuna. 2014. Dodging stress with a personalized biofeedback game. In Proceedings of the first ACM SIGCHI annual symposium on Computer-human interaction in play. ACM, 399400.

2. Miguel Bruns Alonso, David V Keyson, and Caroline Hummels. 2008. Squeeze, rock, and roll; can tangible interaction with affective products support stress reduction?. In Proceedings of the 2nd international conference on Tangible and embedded interaction. ACM, 105-108.

3. Miguel Bruns Alonso, Michel Varkevisser, and David V Keyson. 2012. Expressive stress relievers. In Proceedings of the 7th Nordic Conference on Human-Computer Interaction: Making Sense Through Design. ACM, 761-764.

4. LMG Loe Feijs, GR Geert Langereis, and others. 2010. Designing for heart rate and breathing movements. Design and semantics of form and movement (2010), 57.

5. Anna Mette Fuglseth and Oystein Sorebo. 2014. The effects of technostress within the context of employee use of ICT. Computers in Human Behavior 40 (2014), $161-170$.

6. Diane M Houston and Gillian Marks. 2003. The role of planning and workplace support in returning to work after maternity leave. British Journal of Industrial Relations 41, 2 (2003), 197-214.

7. Janet Ingber. 2016. The Apple Worldwide Developers Conference 2016: What's Coming This Fall. Access WorldMagazine 17, 6 (2016).

8. BS McEwen and T Seeman. 2003. Stress and affect: Applicability of the concepts of allostasis and allostatic load. Handbook of affective sciences (2003), 1117-1137.

9. Phil Parette and Marcia Scherer. 2004. Assistive technology use and stigma. Education and Training in Developmental Disabilities (2004), 217-226.

10. Gina Paul, Barb Elam, and Steven J Verhulst. 2007. A longitudinal study of students' perceptions of using deep breathing meditation to reduce testing stresses. Teaching and learning in medicine 19, 3 (2007), 287-292.

11. Takashi G Sato, Yutaka Kamamoto, Noboru Harada, and Takehiro Moriya. 2013. A playback system that synchronizes the musical phrases with listener's respiration phases. In CHI'13 Extended Abstracts on Human Factors in Computing Systems. ACM, 1035-1040.

12. Peter L Schnall, Paul A Landsbergis, and Dean Baker. 1994. Job strain and cardiovascular disease. Annual review of public health 15, 1 (1994), 381-411.

13. Paul E Spector. 2002. Employee control and occupational stress. Current Directions in Psychological Science 11, 4 (2002), 133-136.

14. Spire. 2017. Spire Breath and activity tracker. Accessed: 2017-06. (2017). https: //www.spire.io/

15. Anna Ståhl, Martin Jonsson, Johanna Mercurio, Anna Karlsson, Kristina Höök, and Eva-Carin Banka Johnson. 2016. The Soma Mat and Breathing Light. In Proceedings of the 2016 CHI Conference Extended Abstracts on Human Factors in Computing Systems. ACM, 305-308.

16. Cati Vaucelle, Leonardo Bonanni, and Hiroshi Ishii. 2009. Design of haptic interfaces for therapy. In Proceedings of the SIGCHI Conference on Human Factors in Computing Systems. ACM, 467-470.

17. Kazuyoshi Wada, Takanori Shibata, Tomoko Saito, and Kazuo Tanie. 2004. Effects of robot-assisted activity for elderly people and nurses at a day service center. Proc. IEEE 92, 11 (2004), 1780-1788. 
18. Steve Yohanan and Karon E MacLean. 2009. A tool to study affective touch. In CHI'09 Extended Abstracts on Human Factors in Computing Systems. ACM, 4153-4158. 